\title{
Functionality of Benecel K4M/Carbopol 971P NF matrices in direct compression tablets for controlled release
}

\author{
Leopoldo Villafuerte-Robles*, Zeltzin Michelet Aguilar-Hernández \\ Departamento de Farmacia, Escuela Nacional de Ciencias Biológicas, Instituto Politécnico Nacional de México, México.
}

\begin{tabular}{|c|c|}
\hline ARTICLE INFO & ABSTRACT \\
\hline $\begin{array}{l}\text { Article history: } \\
\text { Received on: } 16 / 06 / 2016 \\
\text { Revised on: } 17 / 07 / 2016 \\
\text { Accepted on: } 11 / 08 / 2016 \\
\text { Available online: } 26 / 09 / 2016\end{array}$ & $\begin{array}{l}\text { The aim of this work is the assessment of the eventual enhancing effects of Carbopol 971P NF on the } \\
\text { performance of Benecel K4M as a controlled release agent and its impact on other technological properties such } \\
\text { as compactibility and powder flowability. The effect of Carbopol 971P NF and Benecel K4M in the } \\
\text { performance of metronidazole tablets with controlled release was assessed using dissolution and compactibility } \\
\text { profiles and the flowability of powders. Benecel K4M produces release profiles with an average exponent }\end{array}$ \\
\hline $\begin{array}{l}\text { Key words: } \\
\text { Compactibility, Powder } \\
\text { flowability, Compressibility } \\
\text { index, Dissolution profile, } \\
\text { Metronidazole. }\end{array}$ & $\begin{array}{l}\text { parts of Carbopol } 971 \mathrm{P} \text { NF and Benecel K4M was an average of } \mathrm{n}=0.947 \text {. Metronidazole tablets containing the } \\
\text { Benecel K4M/Carbopol } 971 \mathrm{P} N \mathrm{NF} \text { blend shows a compactibility } 2-3 \text { times higher than tablets containing only } \\
\text { Benecel K4M. Metronidazole/Benecel K4M blends flow sufficiently at all studied polymer proportions }(\leq 30 \%) \\
\text { while admixtures of metronidazole/Benecel K4M with Carbopol } 971 \mathrm{P} \text { NF flow sufficiently only at polymer } \\
\text { proportions } \leq 17 \% \text {. Carbopol } 971 \mathrm{P} \mathrm{NF} \text { enhances the overall performance of Benecel K4M in the same way as } \\
\text { Noveon AA1 does, it reduces better the drug release and improves the compactibility, although decreases the } \\
\text { flowability. }\end{array}$ \\
\hline
\end{tabular}

\section{INTRODUCTION}

The functional performance of tablet excipients is related to their physical, chemical and technological properties. These properties allow us to predict whether a particular excipient is likely to have the functionality required to produce a product that meets the specifications of the finished product in all respects (Díaz-Ramírez and Villafuerte-Robles, 2010). The functionality of the excipient can help determine whether or not a drug succeeds or fails (Monsuur and Poncher, 2010). The functional performance of excipients to control the release from drug delivery systems allows the knowledge of their possible uses (Villafuerte-Robles, 2011).Agents to control the release of drug from a tablet ideally provide not only the property to modulate the dissolution rate, but also improve the flow properties of the powders and the compactibility.

\footnotetext{
* Corresponding Author

Leopoldo Villafuerte-Robles, Instituto Politécnico Nacional De México, México.Email: lvillarolvillaro@ hotmail.com
}

The required properties that define the quality of tablets are produced through control of variables such as flowability and tabletability of the mix (Barrios-Vazquez and Villafuerte-Robles, 2013). The Carr's compressibility index (CI) is commonly used as marker of powder flowability (Nalluri and Kuentz, 2010). Before compressing multiparticulate systems in a sustained release dosage form various parameters such as the powder flow properties and compactibility of the powders must be in an acceptable range (Khandai et al., 2014). Hydroxypropyl methylcellulose (HPMC) is a polymer that improves powder system flowability while maintaining compressibility, tablet hardness, and controlled release performance. It helps improve processing speed and predictable and consistent drug release profiles (Introducing Methocel, 2008). Systems containing HPMC produce mostly release profiles ranging from Fickian diffusion to anomalous transport (Juárez et al., 2001; Siepman and Peppas, 2012). Dissolution profiles of niacin from tablets containing different batches of HPMC showed values of the exponent $n$ ranging from 0.69 to 0.76 , indicating combined drug release mechanisms of diffusion and dissolution/erosion of the polymer (Zhou et al., 2014). 
Some other drugs and HPMC polymer grades have observed similar results (Gafourian et al., 2007). The release profiles obtained with HPMC show commonly a burst effect. This means an initial large quantity of the drug is released before the release rate reaches a stable profile. Mostly, the burst effect has been studied to prevent it from occurring in controlled release formulations. Ideally, a controlled release system could be processed in a single step to include a high drug loading and have no burst effect (Huang and Brazel, 2001).

Noveon AA-1 is an acrylic acid polymer of high molecular weight cross-linked with divynil glycol showing bioadhesive and controlled release properties. Noveon AA-1 has binding properties that provide good mechanical properties of tablet formulations. Noveon AA1 hygroscopicity demands processing with low relative humidity. Noveon AA1 has a very fine particle size and static loads, showing no free flowing behavior, segregation can occur in powder blend (Lubrizol, 2014).

Noveon AA1 enhances the overall controlled release performance of HPMC. It induces zero-order release patterns without lag or burst effects, reducing the drug release more efficiently. Noveon AA1 also improves the compactibility, however, decreasing the flowability (Laguna-López and Villafuerte-Robles, 2014). Being Carbopol 971P NF a much less expensive although chemically similar material is the aim of this work the assessment of its eventual similar enhancing effects on the performance of Benecel K4M as a controlled release agent and its impact on other technological properties such as compactibility and powder flowability.

Carbopols are, as Noveon AA1, synthetic high molecular weight polymers of acrylic acid. Carbopol 971P NF is used as controlled release agent and/or binder. In presence of cationic salts, the drug release rate is accelerated and the bioadhesive properties are reduced. Its powder is fluffy and hygroscopic, typically with an equilibrium moisture content of $8-10 \%$ (Rowe $e t$ al., 2009).

Carbopol produces in many cases a zero-order release mechanism. The release mechanism seems to be dependent on the polymer proportion and other added excipients. The use of different proportions of Carbopol 974P produced ibuprofen release profiles with different degrees of curvature. The addition of excipients such as lactose, cellulose and starch changed release profiles towards higher degrees of curvature (Khan and Zhu, 1998).The use of increasing proportions of Carbopol 934P to restrict the release rate of ibuprofen showed increasing values of the exponent indicative of the release mechanism $(n)$ ranging from 0.63 to 0.98 (Khan and Zhu, 1999). This was attributed to reduction of the drug release rate as the polymer content increased, resulting in a longer time to release a given quantity of the drug. This longer time allowing a greater hydration and relaxation of the polymer matrix before this given quantity of drug is released, shifting the release mechanism toward relaxation-erosion, near an apparent order zero release (Tapia-Albarran and Villafuerte -Robles, 2004).

\section{MATERIALS AND METHODS}

\section{Materials}

The materials used in this study were Carbopol 971P NF, obtained from Lubrizol, CDMX (MEXICO); Hydroxypropyl methylcellulose (HPMC), Benecel K4M, obtained from Ashland Specialty Ingredients, CDMX (MEXICO); metronidazole obtained from Química Alkano, CDMX (MEXICO). The drug and the excipients were used as received.

\section{Powders to be processed}

The drug and excipients were evaluated as mixtures. Corresponding amounts of the materials were weighed to obtain $60 \mathrm{~g}$ of mixtures of metronidazole with different proportions of Carbopol 971P NF in a range from $7.5 \%$ to $30 \%$, Benecel $\mathrm{K} 4 \mathrm{M}$ in the same range and a mixture of equal parts thereof ranging from $10 \%$ to $30 \%$, The powders were transferred into a mortar and then mixed for $30 \mathrm{~min}$ in a circular manner with pestle.

\section{Compressibility index - Bulk and tapped density}

The equipment used to assess the powders densities is of our own fabrication and similar to that used to determine the tap density of powders, described in the Handbook of pharmaceutical excipients (Kibe, 2000; Villafuerte Robles, 2001). The tapper was adjusted at a rate of 60 taps per minute and the graduated cylinder was elevated up to a height of $15 \mathrm{~mm}$. This device uses a $100 \mathrm{~mL}$ graduated cylinder joined to a glass funnel with an orifice of 8.6 $\mathrm{mm}$. The bulk $\left(\rho_{b}\right)$ and tapped density $\left(\rho_{t}\right)$ of powders were determined using the above-mentioned tapping machine $(\mathrm{n}=5)$. The $100 \mathrm{~mL}$ measuring cylinder was filled with $60 \mathrm{~g}$ of sample. The volumes were recorded at the beginning (bulk volume) and after 10 taps. The process continued until three successive volume measurements remained constant (tapped volume). The bulk density was calculated as the ratio of mass and bulk volume while the tapped density as the ratio of mass and tapped volume. The registered results are the average of five repetitions with the same sample. The powders were sieved through a number 20 mesh after each repetition. The Carr's Index or compressibility index (CI-\%) was calculated according to the following equation (Mehta et al., 2012):

$$
\text { Compressibility Index }=\frac{\rho t-\rho b}{\rho t} * 100 \quad \text { Eq. } 1
$$

\section{Powders flow rate}

The material is weighed (approximately $30 \mathrm{~g}$ ) and its flow rate assessed using the equipment described to assess the powders densities. The sample is gently poured into the funnel whose bottom opening was blocked. While unlocked, the tapper starts the movement. The powder flows through the orifice of the funnel, falling to the bottom of the cylinder. The time it takes to move the total powder poured through the funnel is registered. The flow rate is calculated by dividing the sample mass by the time. The assay is repeated 5 times, sieving the powder through a mesh number 20 after each measurement. The average of the repetitions is taken as the flow rate. 


\section{Compactibility}

Tablets weighing $200 \mathrm{mg}$ were compacted for $10 \mathrm{~s}$ in a hydraulic press, at a series of compaction pressures from $50 \mathrm{MPa}$ to $170 \mathrm{MPa}$, using $8 \mathrm{~mm}$ circular flat-shaped punch and die. Tablet crushing strength was measured in triplicate, registering the results as an average. For this purpose, a tablet hardness tester Erweka TBH30 was used. The procedure involved placing each tablet diametrically between two flat surfaces and applying pressure until the tablet breaks down. The results were expressed as compactibility curves representing the tablet hardness against the compaction pressure used to obtain the tablets.

\section{Dissolution test}

Tablets obtained as described in the subtitle compactibility and compacted at a compaction pressure of 109 MPa were used to determine the dissolution behavior. The dissolution profile was carried out in a Hanson Research SR6 under sink conditions, for a period of 6 hours using a paddle apparatus at $50 \mathrm{rpm} .900 \mathrm{ml}$ of distilled water was used as the dissolution medium. For each composition, a dissolution test was performed with three repetitions at $37{ }^{\circ} \mathrm{C}$. Each tablet was placed in a coil of stainless steel wire, to prevent sticking or floating. At predetermined time intervals samples were removed, filtered and evaluated with a spectrophotometer (Beckman DU 650, $\lambda=319$ $\mathrm{nm})$. After each sample was removed, the same amount of liquid was replaced, thus maintaining the volume in the vessel. The dissolution profile was expressed as the percentage of drug released, based on the total tablet content after dissolution in a magnetic stirring device.

\section{RESULTS AND DISCUSSION}

\section{Effect of Bencel K4M and Carbopol 971P NF polymers on the release profile}

Drug release from swellable matrices is mainly controlled by polymer water uptake, gel layer formation and polymeric chain relaxation. These parameters are currently regarded as primarily involved in the modulation of drug release.

The use of polymers such as Carbopol 971P NF and Benecel K4M display drug release mechanisms where water molecules tend to diffuse into these systems causing swelling which results in diffusion of drug molecules out through the swollen polymeric matrix. Drug dissolution from solid dosage forms is described with kinetic models in which the amount of drug dissolved $(M)$ is a function of test time $(t)$. In most cases, there is no theoretical concept and some empirical equations have proven to be best suited (Costa and Sousa-Lobo, 2001). In this case, the kinetics of drug release was evaluated with the aid of the exponential or Korsmayer-Peppas model (Eq. 2), which is frequently used for the evaluation of drug release from matrix systems.

$$
M_{t}=\text { Drug released }=K * t^{n} \Leftrightarrow \ln M t=\ln K+n * \ln t \quad \text { Eq. } 2
$$

Usually, the first part of the equation is divided $M \infty$, however, we omitted this term by expressing drug release as a fraction or percentage, $n$ is an exponent indicative of the release mechanism and $K$ is a drug release constant incorporating structural and geometric characteristics of the dosage form. From another point of view, $\ln K$ is the intercept of a linear relationship between the logarithm of the percentage of drug dissolved $(\ln M t)$ and the logarithm of the test time $(\ln t)(\mathrm{Eq}, 2) . K$ means the drug dissolved after $1 \mathrm{~min}$ or the drug dissolved at $\ln \mathrm{t}=0 . K$ values can be considered as an indication of a burst or lag time effect.

One major assumption of the aforementioned model is that the polymer matrix does not swell. Even if swelling does take place in many polymer carriers. However, these models are normally used to investigate a proper "fit" for the experimental measurements of drug release (Razavilar and Choi, 2013).

Figure 1 depicts the release profile of metronidazole tablets containing $30 \%$ of individual polymers or a polymer blend of Carbopol 971P NF and Benecel K4M. The experimental data were treated according to the power law or exponential equation to obtain the depicted regression lines. All experimental data were used for the linear regression analysis.

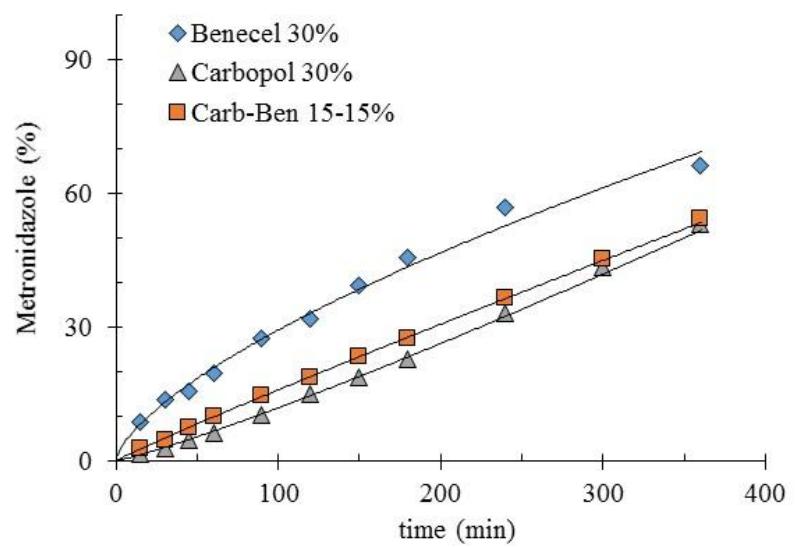

Fig. 1: Release profile of metronidazole matrix tablets with a total polymer content of $30 \%$ of Benecel K4M, Carbopol 971P NF or a mixture of equal proportions thereof. Experimental points and calculated regressions.

The release curves depicted in Figure 1 show a burst release by Benecel $\mathrm{K} 4 \mathrm{M}$ matrices. Burst release is a high rate release of the drug at the beginning of the controlled release process. The burst release is caused by a direct dissolution of the drug without control of the polymeric gel layer. A high $K$ value stands for a burst effect. Benecel matrices display an average $K$ value of 1.87 . This type of matrices show exponent $n$ values smaller than 1.0. This occurs because the drug dissolution is faster than the polymer hydration and the following binding of the particles. Once the gel layer has been established the release rate decreases. This can be considered a deviation of the target zero order sustained release.

On the other hand, Carbopol 971P NF matrices show the opposite, a faster hydration of the polymer producing rapidly a gel layer that control the release rate (Figure 1). It takes some time to the drug to begin to diffuse through the hydrated gel layer. These matrices display a lower $\mathrm{K}$ value, an average of 0.08 . Further, this faster hydration also contributes to a faster swelling, relaxation 
and/or polymer erosion inducing a release rate faster than that of zero order kinetics (exponent $n$ values greater than 1.0).

Carbamazepine mucoadhesive tablets have observed similar dissolution behavior. Matrices containing 75\% HPMC (Benecel) as the main constituent showed a drug release mainly due to diffusion mechanism while tablets containing $75 \%$ Carbopol as the main constituent displayed a drug release governed by the relaxation rate of polymeric chains. Carbopol tablets displaying a lower drug release rate, compared to Benecel tablets (Perioli and Pagano, 2013).

As can be observed in Figure 1, the admixture of Benecel K4M and Carbopol 971P NF produce an intermediate release mechanism, displaying values close to 1.0. This means a near zero order release profile. Carbopol 971P NF is able to induce a zero order release of Benecel $\mathrm{K} 4 \mathrm{M} /$ metronidazole matrices and viceversa, Benecel $\mathrm{K} 4 \mathrm{M}$ is able to induce a zero order release of Carbopol 971P NF/metronidazole matrices.

A similar behavior has been observed by metronidazole matrices containing $10 \% \mathrm{HPMC}$, displaying release profiles with $n$ values of 0.48 that gradually increase up to 1.0 as an increasing proportion of Noveon AA1 is admixed to HPMC. However, matrices containing only Noveon AA1 produce a zero order release kinetics ( $n$ values close to 1.0) and not $n$ values corresponding to a SuperCase II ( $n$ values greater than 1.0) as Carbopol 971P NF does (Laguna-López et al., 2015).

Carbopol 971P NF is a synthetic high molecular weight polymer of acrylic acid that is cross-linked with either allyl sucrose or allyl esters of pentaerythritol. Carbopol 971P NF does not dissolve but swell to a remarkable extent. Different types of Carbopol are available commercially and vary in their chemical structure, degree of cross-linking and residual components. Likewise, Noveon AA1 (polycarbophil) is an acrylic acid polymer of high molecular weight but cross-linked with divynil glycol, showing bioadhesive properties (Rowe et al., 2009).

Zero order release is frequently the target of drug delivery and different methods have been undertaken to design drug delivery vehicles in order to achieve the desired release profile. One of these methods can be the use of the abovementioned polymers, Carbopol 971P NF and Benecel K4M, which produce counteracting effects. As can be seen in Figure 1, the deviations from a zero order kinetics are avoided by the combination of both polymers, one counteracting the effect of the other one.

Tables 1-3 summarize the regression parameters of release curves from metronidazole tablets containing different proportions of Carbopol 971P NF, Benecel K4M or a mixture of equal proportions thereof.

As expected, an increasing proportion of polymers produced slower release rates. Taking as a reference the drug released after $120 \mathrm{~min}$, Benecel K4M matrices release lesser quantities of metronidazole as the polymer proportion increases progressively from $7.5 \%$ to $30 \%$ (Figure 2). In the same way, Increasing proportions of Carbopol 971P NF produce slower release rates. However, the drug released is much smaller than that released from Benecel K4M matrices, less than a half. Further, the drug released stabilizes after a Carbopol 971P NF proportion of $20 \%$. Higher proportions of Carbopol 971P NF do not reduce further, in an important manner, the drug released. Mixtures of both polymers display a behavior similar to Carbopol 971P NF but releasing metronidazole in proportions in between of matrices containing the individual polymers.

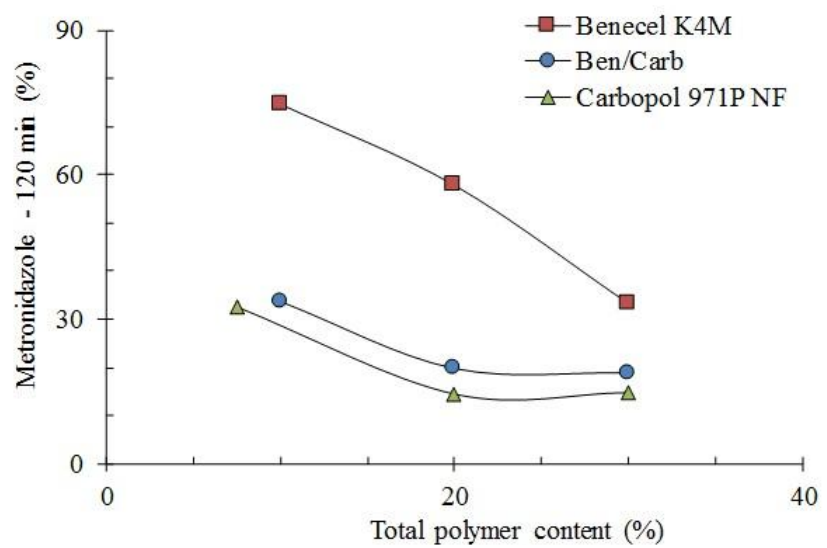

Fig. 2: Effect of polymer proportion on metronidazole release after $120 \mathrm{~min}$, from tablets containing Benecel K4M, Carbopol 971P NF or a mixture of equal proportions thereof.

The $n$ values in the exponential model are considered indicative of the release mechanism. If they range from 0.5 to 1.0 , they are indicative of an anomalous behavior, corresponding to coupled diffusion/relaxation. Values near to 0.5 correspond to a $\mathrm{t}^{1 / 2}$ dependence, generally referred as Fickian release. Values near to 1.0 represent the case-II transport that is purely relaxation controlled. When the value of $n$ is greater than that of the case-II transport $(n>1.0)$, the release is said to be Super case-II transport controlled by polymer dissolution and/or erosion (Brazel and Peppas, 2000; Tapia-Albarran and Villafuerte-Robles, 2004). In the case of a matrix with cylinder form, $n$ is said to be 0.45 instead of 0.5 and 0.89 instead of 1.0 (Kim and Fassihi, 1997).

Drug release from hydrophilic matrices has been attributed to polymer hydration and dissolution (relaxation/erosion mechanism) and to drug diffusion through the gel layer. Different drugs releasing from HPMC matrices showed $n$ values between 0.5 and 1.0 (Martínez-Gonzalez and Villafuerte-Robles, 2003).On the other hand, increasing amounts of Carbopol 974P NF have been observed to result in a reduction of drug release rate and linearization of the release curve, leading to a shift from anomalous type of release towards a swelling controlled case-II mechanism (Khan and Zhu, 1998).

The regression parameters from Carbopol 971P NF tablets (Table 1) indicate a release mechanism corresponding with a process controlled by relaxation and disentanglement or erosion of the polymeric matrix, $n$ values $>1.0$. On the other hand, matrix tablets releasing metronidazole from Benecel $\mathrm{K} 4 \mathrm{M}$ matrices display $n$ values corresponding with an anomalous process, $n$ values between 0.5 and 1.0 (Table 2). This meaning the release mechanism is controlled by diffusion and relaxation. 
Table 1: Calculated regression parameters of metronidazole release profiles from tablets containing different proportions of Carbopol 971P NF, according to the exponential model. $\mathrm{Mt}=\mathrm{K} * \mathrm{t}^{\mathrm{n}}$.

\begin{tabular}{cccc}
\hline Carbopol 971P NF & Release constant & Exponent & $\mathbf{r}^{2}$ \\
\hline$(\%)$ & $(K)$ & $(n)$ & \\
7.5 & 0.158 & 1.11 & 0.98 \\
10 & 0.076 & 1.19 & 0.99 \\
20 & 0.026 & 1.32 & 0.99 \\
30 & 0.060 & 1.15 & 0.99 \\
\hline
\end{tabular}

Table 2: Calculated regression parameters of metronidazole release profiles from tablets containing different proportions of Benecel K4M, according to the exponential model. $\mathrm{Mt}=\mathrm{K} * \mathrm{t}^{\mathrm{n}}$.

\begin{tabular}{cccc} 
exponent K4M & Release constant & Exponent & $\mathbf{r}^{2}$ \\
\hline Benecel & $(K)$ & $(n)$ & \\
7.5 & 1.71 & 0.724 & 0.99 \\
10 & 3.08 & 0.666 & 0.99 \\
20 & 1.37 & 0.782 & 0.92 \\
30 & 1.34 & 0.670 & 0.99 \\
\hline
\end{tabular}

Table 3: Calculated regression parameters of metronidazole release profiles from tablets containing different proportions of a mixture of equal proportions of Carbopol 971P NF and Benecel K4M, according to the exponential model. $\mathrm{Mt}=\mathrm{K} * \mathrm{t}^{\mathrm{n}}$.

\begin{tabular}{cccc}
\hline $\begin{array}{c}\text { Carbopol 971P } \\
\text { NF/Benecel K4M }\end{array}$ & Release constant & Exponent & $\mathbf{r}^{\mathbf{2}}$ \\
\hline$(\%)$ & $(K)$ & $(n)$ & \\
10 & 0.241 & 1.032 & 0.99 \\
15 & 0.283 & 0.905 & 0.99 \\
20 & 0.261 & 0.904 & 0.99 \\
30 & 0.204 & 0.946 & 0.99 \\
\hline
\end{tabular}

Oral prolonged release delivery systems are supposed to provide a slow and continuous release rate of the drug, limiting the entrance of the drug to the systemic circulation. To maintain optimum bioavailability, it is important that the fraction of drug dose released from a drug delivery system is sufficiently large to make for drug metabolized and/or excreted during the same period. This is why a zero order release is desirable. Ideally, a desirable drug delivery system should be one that can deliver or release the drug molecules at a constant rate, with the body acting as a perfect sink.

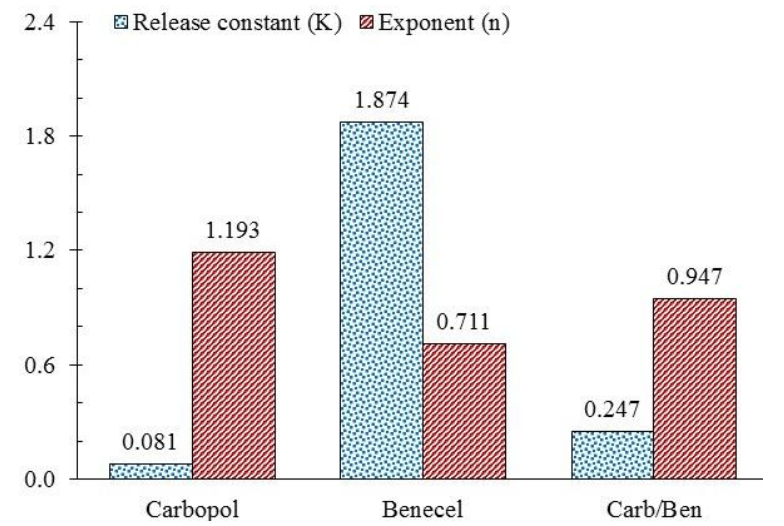

Fig. 3: Effect of polymer type on the average values of regression parameters of metronidazole release profiles from tablets.

As can be seen in Figure 3, a mixture of equal parts of both polymers, Benecel K4M and Carbopol 971P NF, produce a release kinetics close to order zero, $n=0.947$. This release kinetics satisfying not only the drug delivery but also an almost lineal release from the very beginning; practically no burst effect and no lag time in the dissolution process (Figure 1).

Similar results have been observed by buccal compacts of miconazole containing Carbopol $71 \mathrm{G}$ and /or Noveon AA1. The compacts displayed release profiles with a low degree of curvature and with no apparent lag or burst effects (Mohamed, 2011). A chitosan-polycarbophil complex formulated as controlled release tablets of 5-fluorouracil showed also similar release profiles with a low degree of curvature and without burst effect (Pendekal and Tegginamat, 2012).

\section{Effect of Benecel K4M and Carbopol 971P NF polymers on compactibility of metronidazole tablets}

Selection of excipients requires a balance between efficiency in time, cost and performance expected in the product. Excipients are included in a formulation by their properties, which together with the processes allow the production of dosage forms with the required specifications. In this sense, tablets must have sufficient strength to resist wear or breakage during handling or processing, particularly during packing. For this reason, the hardness, as a specification of the tablets, is important and with practical relevance.

Figure 4 depicts the compactibility profile of metronidazole tablets containing $20 \%$ of Benecel K4M, Carbopol 971P NF or a mixture of equal proportions thereof. Compactibility profiles of metronidazole tablets added of $20 \%$ Benecel K4M show the lowest tablet hardness in a range up to $25 \mathrm{~N}$. Carbopol 971P NF improves the compactibility of Benecel K4M admixtures in an important manner up to $63 \mathrm{~N}$ because of the high compactibility of Carbopol 971P NF. Carbopol 971P NF shows in admixtures with metronidazole tablet hardness values in a range up to $95 \mathrm{~N}$. Compactibility curves describe the relationship between hardness or tensile strength of the tablets and the compaction pressure used to obtain them. Figure 4 describes the experimental points and the calculated regressions with a logarithmic model.

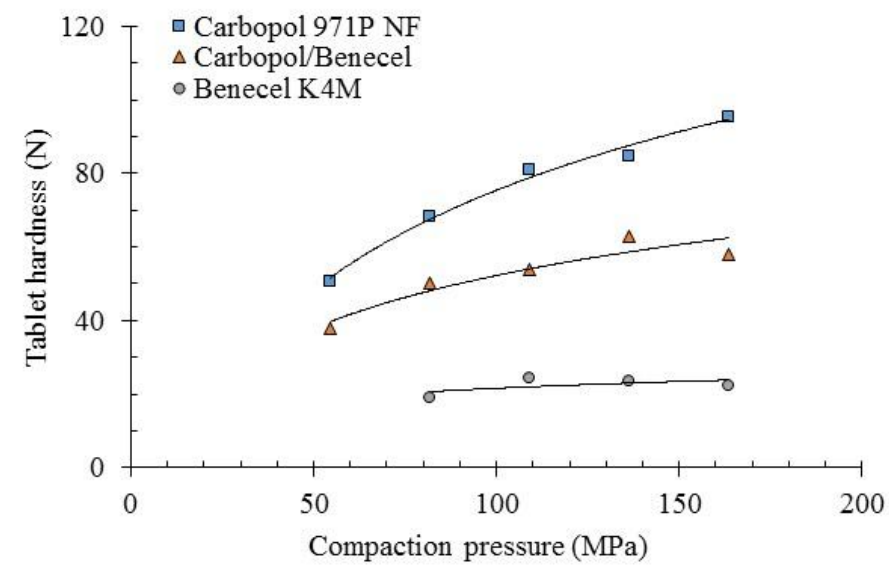

Fig. 4: Compactibility profiles of metronidazole tablets containing $20 \%$ of Benecel K4M, Carbopol 971P NF or a mixture of equal proportions of both polymers. 
Taking as a reference the tablet hardness obtained with tablets compacted at $136 \mathrm{MPa}$, Figure 5 depicts the effect of polymer proportion on compactibility of admixtures of metronidazole with the different polymers. The tablet hardness of admixtures of metronidazole with Benecel K4M increases almost three-fold after admixture with Carbopol 971P NF.

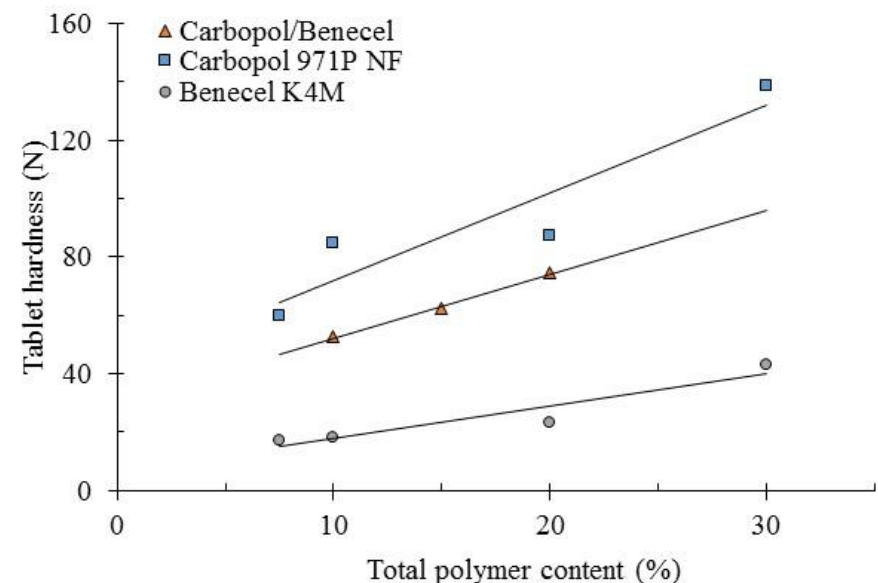

Fig. 5: Effect of polymer proportion on the calculated tablet hardness, at $\mathrm{Pc}$ $=136 \mathrm{MPa}$, of metronidazole tablets containing Benecel K4M, Carbopol 971P $\mathrm{NF}$ or a mixture of equal proportions of both polymers.

According to the slope of the regression lines depicted in Figure 5, the compactibility of metronidazole tablets containing Benecel K4M shows a tablet hardness increase of $1.1 \mathrm{~N}$ per unit percentage of added excipient while tablets containing the admixture of Carbopol 971P NF with Benecel K4M increases the tablet hardness by a factor of $2.2 \mathrm{~N}$. This factor is the $3.0 \mathrm{~N}$ by tablets containing only Carbopol 971P NF. Carbopol 971P NF improves the functionality of Benecel $\mathrm{K} 4 \mathrm{M}$ as a direct compression agglutinant in an important manner.

\section{Effect of Benecel K4M and Carbopol 971P NF polymers on flowability of metronidazole powder blends}

Powder flow is critical during tableting, as powders must flow easily and uniformly into the tablet dies to ensure tablet weight uniformity and tablets with consistent and reproducible properties. Interparticle forces and the packing structure mainly determine the behavior of powders. A manner to determine the powder flowability is using the compressibility index (CI). The compressibility index is inversely proportional to flowability of powders. Higher compressibility index values corresponding with higher interparticle forces and/or denser packing.

It has been observed high compressibility index values in formulations containing 20\% of Carbopol 934 and 974 (32\%). Further, these formulas do not flow at all through a funnel with an opening of $10 \mathrm{~mm}$. It indicates a poor powder flowability and the need of glidants to make them processable (Stanescu et al., 2010). Compared to some other excipients Carbopol can be considered dysfunctional in this respect. Compressibility index of microcrystalline cellulose type 102 (Helmcel 200) is $21.7 \%$, indicating a passable powder flowability while the compressibility index of agglomerated isomaltose (GalenIQ 720) was determined to be $13.8 \%$, indicating a good powder flowability (FuentesGonzalez and Villafuerte-Robles, 2014).

Figure 6 depicts the change in compressibility index of metronidazole blends after addition of different proportions of Carbopol 971P NF, Benecel K4M and a mixture thereof (50:50). It is observed a progressive increase in compressibility index as the proportion of polymers increases, which stands for a decrease in flowability. According to the calculated regression lines, Carbopol 971P NF increases progressively the compressibility index of metronidazole in their blends, in about $0.73 \%$ per percentage point of added excipient; showing a CI of $20.8 \%$ at a Carbopol 971P NF proportion of $7.5 \%$. This lesser proportion of Carbopol 971P NF indicates a powder flowability considered as processable. Similar results have been observed by formulations of gastric floating tablets containing timolol maleate. Carbopol 971P NF proportions between $6 \%$ and $9 \%$ showed a compressibility index of about $19 \%$ (Charyulu-Narayana et al., 2013).

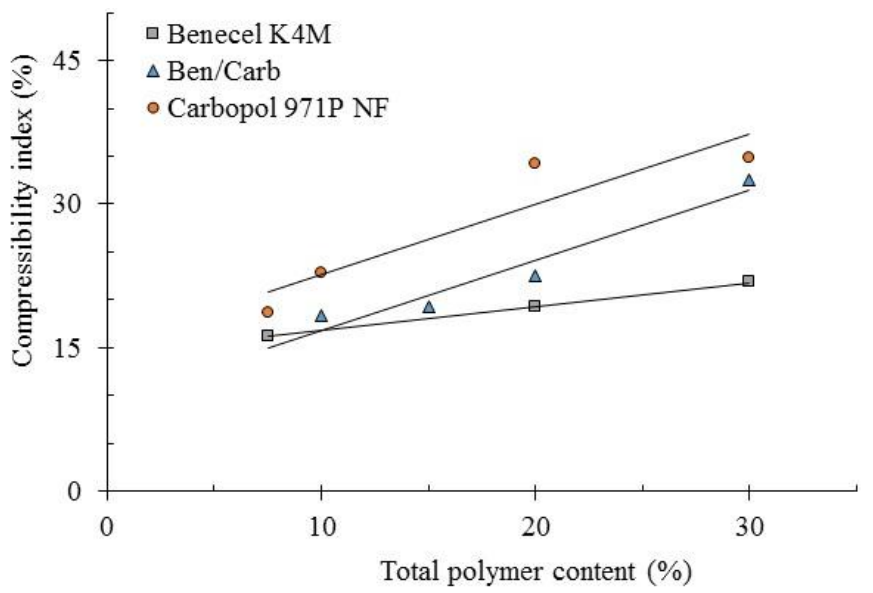

Fig. 6: Effect of polymer proportion on the compressibility index of metronidazole mixtures containing Benecel K4M, Carbopol 971P NF or a mixture of equal proportions of both polymers.

In the same way, addition to metronidazole of increasing proportions of the blend of Carbopol 971P NF and Benecel K4M increases also progressively the powders compressibility index in $0.73 \%$ per percentage point of added polymers mixture. Even if, the polymers blend shows lesser CI values (14.9\%) than Carbopol 971P NF alone $(20.8 \%)$. The mixing of Carbopol 971P NF with Benecel K4M decreases in about $28 \%$ the powders compressibility index displayed by Carbopol 971P NF alone. Further, it is observed a lesser increase of CI when the added polymer is Benecel K4M. Benecel K4M increases the CI of metronidazole in only $0.25 \%$ per percentage point of added excipient, about a third of the increase showed by Carbopol 971P NF. Benecel K4M mixtures with metronidazole display a compressibility index in a range of $16.2-21.8 \%$.

Considering that microcrystalline cellulose type 102 $(\mathrm{CI}=21.7 \%)$ is at the limit of processability of powders in a highspeed tableting machine. The compressibility index suggests that the blend of metronidazole/Carbopol 971P NF is processable in 
such type of tableting machines only at Carbopol 971P NF proportions $\leq 9 \%$. Admixture of Benecel K4M allows the use of polymer proportions $\leq 17 \%$. Benecel K4M mixtures are sufficiently flowable at all studied polymer proportions $(\leq 30 \%)$. The above-mentioned results are confirmed by the powder flow rate through a glass funnel with an opening of $8.6 \mathrm{~mm}$. Figure 7 depicts the effect of polymer proportion on the powder flow rate of metronidazole mixtures containing different proportions of Benecel K4M, Carbopol 971P NF or a mixture of equal proportions of both polymers. Carbopol 971P NF mixtures with metronidazole display calculated powder flow rates between 0.06 $\mathrm{g} / \mathrm{s}$ and $0.24 \mathrm{~g} / \mathrm{s}$, followed by the mixtures of Carbopol 971P $\mathrm{NF} /$ Benecel K4Mwhich display powder flow rates up to $1.14 \mathrm{~g} / \mathrm{s}$. It means that Carbopol 971P NF powder flow rate betters more than fourfold when admixed with Benecel K4M. On the other hand, Benecel K4M mixtures flow freely in a range from $3.7 \mathrm{~g} / \mathrm{s}$ to 6.7 $\mathrm{g} / \mathrm{s}$.

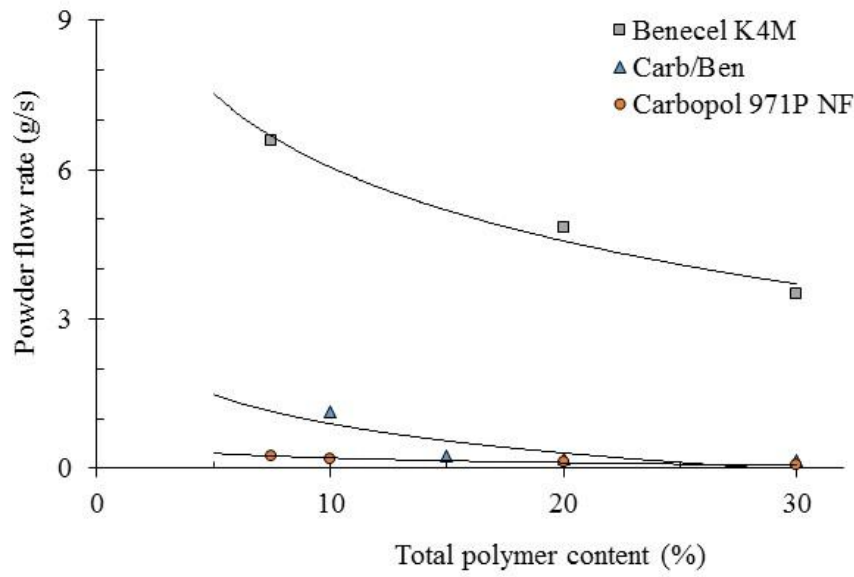

Fig. 7: Effect of the total polymer content on the powder flow rate of metronidazole mixtures containing different proportions of Benecel K4M, Carbopol 971P NF or a mixture of equal proportions of both polymers.

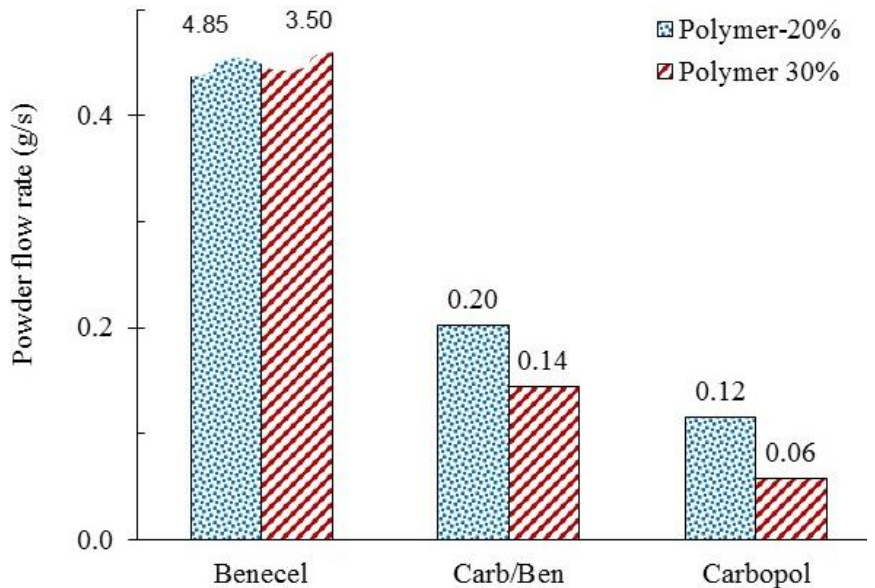

Fig. 8: Effect of $20 \%$ and $30 \%$ total polymer content on the powder flow rate of metronidazole mixtures containing Benecel K4M, Carbopol 971P NF or a mixture of equal proportions of both polymers.

A comparative view of the effect of Carbopol 971P NF, Benecel $\mathrm{K} 4 \mathrm{M}$ and a mixture thereof on the powder flow rate of metronidazole is depicted in Figure 8 when using polymer proportions of $20 \%$ and $30 \%$. The admixture of Benecel K4M to Carbopol 971P NF increases the Carbopol 971P NF flow rate to the double.

\section{CONCLUSION}

Carbopol 971P NF enhances the functionality of Benecel $\mathrm{K} 4 \mathrm{M}$ as a controlled release agent used for direct compression in a similar way as Noveon AA1 does. Carbopol 971P NF modify the Benecel K4M release behavior predominantly controlled by an anomalous transport process towards a zero-order release kinetics. Further, the initial burst effect due to the slow hydration of Benecel K4M is avoided after admixture with Carbopol 971P NF. The Benecel K4M efficiency to reduce the drug release rate is also enhanced with Carbopol 971P NF; the polymers mixture reduces the release rate more than the double compared to Benecel K4M. The admixture of Carbopol 971P NF improves the overall efficiency of the Benecel $\mathrm{K} 4 \mathrm{M}$ controlled release performance with a release pattern close to order zero. As a direct compression agglutinant, Carbopol 971P NF also improves the functionality of Benecel K4M. Metronidazole/Benecel K4M tablets display compactibilities about the double of those tablets containing only Benecel K4M. The admixture of Carbopol 971P NF increases importantly the compactibility of metronidazole/Benecel K4M tablets. Contrasting, Carbopol 971P NF affects negatively the rheological properties of metronidazole/Benecel K4M combinations. The use of Carbopol 971P NF deteriorates the the flowability of metronidazole/Benecel K4M powder blends. Metronidazole/Benecel K4M mixtures are sufficiently flowable at all studied polymer proportions $(\leq 30 \%)$ while the addition of Benecel K4M/Carbopol 971P NF admixtures to metronidazole will be flowable only at polymer proportions $\leq 17 \%$. The use of higher proportions of the Benecel K4M/Carbopol 971P NF admixture will require the addition of glidants to make processable the metronidazole powder blends.

\section{Financial support and sponsorship: Nil.}

Conflict of Interests: There are no conflicts of interest.

\section{REFERENCES}

Barrios-Vazquez SC, Villafuerte-Robles L. Functionality of GalenIQ 721 as excipient for direct compression tablets. J App Pharm Sci, 2013; 3: 8-19.

Brazel CS, Peppas NA. Modeling of drug release from swellable polymers. Eur J Pharm Biopharm, 2000; 49(1): 47-58.

Charyulu-Narayana R, Girish S K, Patil AB. Effect of combination of HPMC and Carbopol 934P on the properties of the gastric floating tablets timolol maleate. J Drug Del \& Therap, 2013; 3(4): 20-5.

Costa P, Sousa-Lobo JM. Modeling and comparison of dissolution profiles. Eur J Pharm Sci; 2001; 13: 123-33.

Díaz-Ramírez CC, Villafuerte-Robles L. Surrogate functionality of celluloses as tablet excipients. Drug Dev Ind Pharm, 2010; 36: 1422-35.

Fuentes-Gonzales KI, Villafuerte-Robles L. Powder fluidity as a functionality parameter of the excipient GalenIQ 720. Int J Pharm Pharmac Sci, 2014; 6 (9): 66-74. 
Gafourian T, Safari A, Adibkia K, Parviz F, Nokhodchi A. A drug release study from hydroxypropylmethylcellulose (HPMC) matrices using QSPR modeling. J Pharm Sci, 2007; 96 (12): 3334-51.

Huang X, Brazel CS. On the importance and mechanisms of burst release in matrix-controlled drug delivery systems. J Control Release, 2001; 73: 121-36.

Introducing Methocel DC Grade hypromellose polymers for direct compression of controlled release dosage forms. October 2008. Available at: http://msdssearch.dow.com/PublishedLiteratureDOWCOM/ dh_01c3/0901b803801c3f17.pdf?filepath=/198-

02173.pdf\&fromPage $=$ GetDoc. [Accessed 21 August 2014].

Juárez H, Rico G, Villafuerte L. Influence of admixed carboxymethylcellulose on release of 4-aminopyridine from hydroxypropyl methylcellulose matrix tablets. Int J Pharm, 2001; 216:11525.

Khan GM., Zhu JB. Formulation and in vitro evaluation of ibuprofen-Carbopol 974P NF controlled release matrix tablets III: influence of co-excipients on release rate of the drug. J Control Release, 1998; 54: 185-90.

Khan GM., Zhu JB. Studies on drug release kinetics from ibuprofen-carbomer hydrophilic matrix tablets: influence of co-excipients on release rate of the drug. J Control Release, 1999; 57: 197-203.

Khandai M., Chakraborty S., Ghosh, AK. Critical analysis of algino-Carbopol multiparticulate system for the improvement of flowability, compressibility and tableting properties of a poor flow drug. Powder Technol, 2014; 253: 223-9.

Kibbe AH. Editor. 2000. Handbook of pharmaceutical excipients, third edition. London, UK: Pharmaceutical Press. pp. 641.

Kim, H., Fassihi, R. Application of binary polymer system in drug release rate modulation. 2. Influence of formulation variables and hydrodynamic conditions on release kinetics. J. Pharm. Sci, 1997; 86: 323-8.

Laguna-López S, Villafuerte-Robles L. Noveon AA1 as enhancer of HPMC as a direct compression matrix for controlled release. J App Pharm Sci, 2014; 4 (11): 62-8.

Laguna-López S, Angulo-Delgado CR, Villafuerte-Robles L. Effect of insoluble excipients on performance of direct compression controlled release matrices. Lat Am J Pharm, 2015; 34 (3): 552-61.

Lubrizol. Guidance document for processing Carbopol polymers in solid dosage forms. Available at: http://www.lubrizol.com/ Life-Science/Documents/Pharmaceutical/Brochures/ Guidance-Documenton-Processing-Carbopol--Polymers-in-Oral-Solid-Dosage-

Forms.pdf.[Accessed 30 April 2014].

Martínez Gonzalez I, Villafuerte Robles L. Effect of varying the restriction degree of 4-aminopyridine release from HPMC matrices on the mechanism controlling the process. Int. J. Pharm, 2003; 257 (1, 2): 253 64.

Mehta, S.; De Beerb, T.; Remon, J.P.; Vervaet, Ch. Effect of disintegrants on the properties of multi-particulate tablets comprising starch pellets and excipient granules. Int J Pharm; 2012; 422: 310-7.

Mohamed SP. Influence of the polymers and co-excipients on the performance of buccal bioadhesive tablets containing miconazole nitrate. The Pharma Res J, 2011; 06 (01):102-11.
Monsuur F, Poncher J. Raising expectations of excipients. Chimica oggi / Chemistry today. Focus on Excipients. September/October, 2010; 28(5): V-VI.

Nalluri VR, Kuentz M. Flowability characterization of drugexcipient blends using a novel powder avalanching method. Eur J Pharm Biopharm, 2010; 74:388-96.

Pendekal MS, Tegginamat PK. Development and characterization of chitosan-polycarbophil interpolyelectrolyte complex based 5-fluorouracil formulations for buccal, vaginal and rectal application. DARU J Pharm Sci, 2012; 20:67.

Perioli, L., Pagano, C. Preformulation studies of mucoadhesive tablets for carbamazepine sublingual administration. Colloids and Surfaces B: Biointerfaces, 2013; 102: 915-22.

Razavilar N, Choi P. In-vitro modeling of the release kinetics of micron and nano-sized polymer drug carriers. Int J Drug Delivery, 2013; 5: 362-78.

Rowe RC, Sheskey PJ, Quinn ME. 2009. Handbook of pharmaceutical excipients, 6th edition. London, UK: Pharmaceutical Press110-4.

Siepmann J, Peppas NA. Modeling of drug release from delivery systems based on hydroxypropyl methylcellulose (HPMC). Adv Drug Del Rev, 2012; 64: 163-74.

Stanescu A, Ochiuz L, Cojocru I, Popovici I, Lupuleasa D. The influence of different polymers on the pharmaco-technological characteristics of propiconazole nitrate bioadhesive oromucosal tablets. Farmacia, 2010; 58 (3): 279-89.

Tapia-Albarran, M., Villafuerte-Robles, L. Assay of amoxicillin sustained release from matrix tablets containing different proportions of Carbopol 971P NF. Int J Pharm, 2004; 273: 121-7.

Villafuerte Robles L. Propiedades reológicas de los polvos farmacéuticos: un nuevo equipo. Rev Mex C Farm, 2001; 32 (1): 11-5.

Villafuerte-Robles L. Los excipientes y su funcionalidad en productos farmacéuticos sólidos. Rev Mex C Farm, 2011; 42(1): 18-36.

Zhou D, Law D, Reynolds J, Davis L, Smith C, Torres JL, Dave V, Gopinathan N, Hernandez DT, Springman MK and Zhou CC. Understanding and Managing the Impact of HPMC Variability on Drug Release from Controlled Release Formulations. J Pharm Sci, 2014; 103 (6): 1664-72.

\section{How to cite this article:}

Villafuerte-Robles L, Aguilar-Hernández ZM. Functionality of Benecel K4M/Carbopol 971P NF matrices in direct compression tablets for controlled release. J App Pharm Sci, 2016; 6 (09): 001008. 